\title{
Dorsal raphe nucleus and harm avoidance: A resting-state investigation
}

\author{
N. Meylakh ${ }^{1}$ L. A. Henderson ${ }^{1}$
}

Published online: 23 March 2016

(C) Springer Science+Business Media New York (outside the US) 2016

\begin{abstract}
The temperament dimension of harm avoidance defines an individual's biological tendency to exhibit altering levels of anxious, inhibiting, and cautious behavior. High harm avoidance and anxiety are highly comorbid, likely due to activity in similar neural circuitries involving the dorsal raphe nucleus. Despite the many investigations that have explored personality factors and brain function, none have determined the influence of ongoing activity within dorsal raphe networks on harm avoidance. The aim of this study was to explore such a relationship. In 62 healthy subjects, a series of 180 functional magnetic resonance images covering the entire brain were collected, and each subject completed the 240-item TCI-R questionnaire. Independent component analyses were performed to define the dorsal raphe network and then to determine the regions significantly correlated with harm avoidance. The independent component analyses revealed three signal intensity fluctuation maps encompassing the dorsal raphe nucleus, showing interactions with regions of the amygdala, hippocampus, nucleus accumbens, and prefrontal, insular, and cingulate cortices. Within these systems, the resting signal intensity was significantly coupled to harm avoidance in the bilateral basal amygdala, bilateral ventral hippocampus, bilateral insula, bilateral nucleus accumbens, and medial prefrontal cortex. Note that we could not measure serotonergic output, but instead measured signal changes in the dorsal raphe that likely reflect synaptic activity. These data provide evidence that at rest, signal intensity fluctuations within the dorsal raphe networks are related to harm avoidance.
\end{abstract}

L. A. Henderson

lukeh@anatomy.usyd.edu.au

1 Department of Anatomy and Histology, F13, University of Sydney, Sydney, NSW 2006, Australia
Given the strong relationship between harm avoidance and anxiety-like behaviors, it is possible that ongoing activity within this identified neural circuitry can contribute to an individual developing anxiety disorders.

Keywords Amygdala $\cdot$ Hippocampus $\cdot$ Anxiety $\cdot$ Functional magnetic resonance imaging

Robert Cloninger's biopsychosocial model conceptualizes personality as an interaction between inherited biological traits and changeable characteristics (Cloninger, Svrakic, \& Przybeck, 1993). The model separates personality into seven dimensions: four temperaments, the "emotional core" of personality, and three character dimensions, evolving as an individual grows and interacts with society. It has been hypothesized that three of the temperament dimensions - harm avoidance, novelty seeking, and reward dependence - are associated with activity in specific neurotransmitter systems. Consequently, dysregulation of these particular systems can predispose an individual to experience abnormalities in their concomitant personality temperaments, and this can predispose the expression of a personality or affective disorder in a stressful environment (Lovinger, 1999).

Harm avoidance (HA) is defined as a "genetically determined bias toward being cautious, apprehensive and overly pessimistic" (Baeken, De Raedt, Ramsey, Van Schuerbeek, Hermes, Bossuyt, \& Luypaert, 2009). Low HA has been associated with risk-taking, harmful behavior, impulsiveness, suicidal ideation, and aggression (Cloninger, 1986; Peirson, Heuchert, Thomala, Berk, Plein, \& Cloninger, 1999), whilst high HA has been associated with behavioral inhibition, anxiety and neuroticism (Carver \& Miller, 2006). HA is referred to as the anxietyrelated personality dimension (Montag, Reuter, Jurkiewicz, Markett, \& Panksepp, 2013), and therefore high HA levels are tightly associated with neurobiological pathologies such as 
depression and anxiety (Arnold, Zai, \& Richter, 2004; Commons, Connolley, \& Valentino, 2003; Kenna, RoderHanna, Leggio, Zywiak, Clifford, Edwards, \& Swift, 2012; Westlye, Bjornebekk, Grydeland, Fjell, \& Walhovd, 2011).

It has been hypothesized that HA levels and anxiety are directly influenced by activity levels within the ascending serotonergic system, which predominantly originates within the dorsal raphe nucleus (DR; Waselus, Valentino, \& Van Bockstaele, 2011). Recent neuroimaging studies support this hypothesis by suggesting that anxiety is regulated by serotonergic activity and that activity within the ascending serotonergic system plays a crucial role in the pathogenesis of anxiety disorders (Vertes \& Linley, 2007; Westlye et al., 2011). Furthermore, high HA has been characterized by high levels of serotonin, which in turns underlies the biology of a highly anxious individual (Montag et al., 2013). Given the proposal that HA traits are biological and directly related to serotonergic activity, it is plausible that HA levels may have significant effects on the resting activity coupling between regions of the ascending serotonergic system. If activity coupling within the serotonergic system is indeed related to HA levels, individuals could be biased to react in a particular manner when placed into a challenging environment, which could predispose them to developing anxiety disorders. The DR could be a good region to provide insight into this system.

The aim of this study was to use resting-state functional magnetic resonance imaging (fMRI) to determine the relationships between the DR and HA. We hypothesized that the DR would be functionally connected to regions of the welldescribed ascending serotonergic system, including the hippocampus and amygdala (Vertes \& Linley, 2007). Furthermore, we hypothesized that the strength of the functional coupling within some of these regions would be significantly correlated with an individual's HA. The results of this study will help us understand how ongoing activity within a particular neural system can influence personality trait expression; which will, in turn, lay the groundwork for exploring the activity of these circuits in individuals with personality and anxiety disorders.

\section{Method}

Sixty-two healthy subjects were recruited. The mean $( \pm S E M)$ age of the subjects was $34.5 \pm 2.2$ years (range $20-65 ; 41$ females, 21 males). Subjects were excluded from the investigation if they had any diagnosed neurological or psychological disorders, were taking any mood-altering medications, or had metal implants or other characteristics that would exclude them from magnetic resonance imaging (MRI) procedures. All procedures were approved by the Human Research Ethics Committees of the University of Sydney, and written consent was obtained from all subjects in accordance with the Declaration of Helsinki.

\section{Psychological measures}

All subjects completed the revised 240-item TCI-R (Cloninger et al., 1993), which assesses the seven dimensions of personality according to Cloninger's biopsychosocial model. Each subject was instructed to answer 240 questions on a 0 to 4 scale ( 0 false, 1 mostly or probably false, 2 neither true nor false, 3 mostly or probably true, 4 true). Each answer was then manually inserted into an installed TCI-R program that calculated the overall score for each subject for each dimension. For the purposes of this study, only HA scores were used for the analysis. The subjects completed the questionnaire within seven days of the MRI scanning session.

\section{MRI acquisition and analysis}

Brain imaging was performed using a 3-T MRI scanner (Philips, Achieva, 32-channel SENSE head coil). Subjects lay supine on the scanner bed with their heads secure in a tight-fitting head coil. With the subject relaxed and with eyes closed, a high-resolution 3-D T1-weighted anatomical image set was collected (turbo field echo; $\mathrm{TE}=2.5 \mathrm{~ms}$, TR $=5$, $600 \mathrm{~ms}$, flip angle $=8^{\circ}$, voxel size $=0.8 \mathrm{~mm}^{3}$ ). This was followed by a resting-state fMRI scan, during which a series of 180 gradient echo-planar fMRI image volumes, using blood-oxygen-level-dependent contrast, was collected. Each image volume contained 35 axial slices covering the entire brain (raw voxel $=3 \times 3 \times 4 \mathrm{~mm}$ thick, repetition time $=2$, $000 \mathrm{~ms}$, echo time $=40 \mathrm{~ms})$.

Using SPM8 (Friston, Holmes, Poline, Grasby, Williams, Frackowiak, \& Turne, 1995), all of the fMRI images were realigned and bias-corrected to remove field inhomogeneities, and global signal drifts were removed using the detrending method described by Macey and colleagues (Macey, Macey, Kumar, \& Harper, 2004). Movement parameters created from the realignment step were visualized, and we noted that no subject displayed movements of greater than $1 \mathrm{~mm}$ in any direction. As a result, all 62 subjects were used for further analysis. The fMRI image sets from each individual were then coregistered to each subject's own T1-weighted anatomical set, which was then spatially normalized to the Montreal Neurological Institute (MNI) template. These normalization parameters were then applied to the fMRI image sets so that both the T1-weighted anatomical and fMRI images sets were in the same location as the MNI template.

Given that we were interested first in defining an extensive neural circuitry that almost certainly receives inputs from multiple sites, and therefore contains multiple signal intensity input patterns, we used an ICA to search for consistent signal intensity change patterns across the subjects. ICA is a 
technique that can extract independent sources of activity within a recorded mixture of sources and can detect underlying factors of a data set that are often lost in other analyses, such as seed-based techniques (Brown, Yamada, \& Sejnowski, 2001; Frigyesi, Veerla, Lindgren, \& Hoglund, 2006). ICA is suited to neural systems analyses because it can detect ongoing activity fluctuations within widespread neural sites with great sensitivity. We performed an ICA with the Group ICA toolbox in SPM8 (Calhoun, Adali, Pearlson, \& Pekar, 2001). Using the Infomax ICA algorithm, we estimated 40 independent component maps in which the signal intensities within multiple regions covaried. To explore those neural networks associated with the DR, we rank-ordered these components spatially with relation to the DR. That is, we created a volume of interest (VOI) restricted to the DR on a mean fMRI image set and then ranked in order those neural networks (components) that included signal intensity fluctuations within the DR. We created the DR VOI on the basis of Duvernoy's (1995) atlas, The Human Brain Stem and Cerebellum, and from an investigation of the cytoarchitecture of the human DR (Baker, Halliday, \& Tork, 1990; see Fig. 1). Using this sorting method, we were able to define three separate signal intensity patterns that were associated with activity within three widespread neural circuits involving the DR.

Using a random-effects procedure, one-sample $t$ tests were then performed on each of the three independent components to determine the brain regions in which signal intensity fluctuations were tightly coupled $(p<.05$, family-wise error corrected for multiple comparisons, minimum cluster extent ten voxels). The relationships between signal intensity fluctuations within the DR networks and HA were then determined by performing correlation analyses between HA and signal intensity fluctuations, with the effects of age and gender removed by including them as nuisance variables. Since we hypothesized that regions within the DR networks would be influenced by
HA, small-volume corrections were applied to each significantly correlated cluster $(p<.05)$. In addition, for each significantly correlated cluster, parameter estimate values were extracted and plotted against individual HA values, and rho values were then calculated and significance determined $(p<.05$, Bonferroni corrected for multiple comparisons, $n=6)$. These parameter estimate values essentially quantify the functional connectivity strength between the brain regions in each network and how they vary with HA. A positive relationship suggests increased connectivity with increasing HA scores, and vice versa. Finally, for each cluster, significant differences in parameter estimate values between those individuals with high HA (HA score $>99$ ) and low HA (HA score $<83$ ) were determined (two-sample $t$ test, $p<.05$, Bonferroni corrected for multiple comparisons, $n=6$ ).

\section{Results}

\section{Psychological measures}

HA scores ranged from 52 to 120 with a mean $( \pm S E M)$ of $91.3 \pm 2.3$. This mean value lies within the range considered to be high average.

\section{Dorsal raphe network}

ICA revealed three components whose signals encompassed the DR (Fig. 2). Signal intensity fluctuations in Component 1 were restricted to the regions of the hypothalamus and insula. Signal intensity fluctuations within Component 2 also encompassed a large extent of the insula, but also included the caudate, putamen, and nucleus accumbens, as well as discrete regions of the anterior cingulate cortex (ACC), medial prefrontal cortex (mPFC), and dorsolateral prefrontal cortex (dlPFC). Finally, Component 3 included the vast majority of the amygdala, hippocampus, and temporal

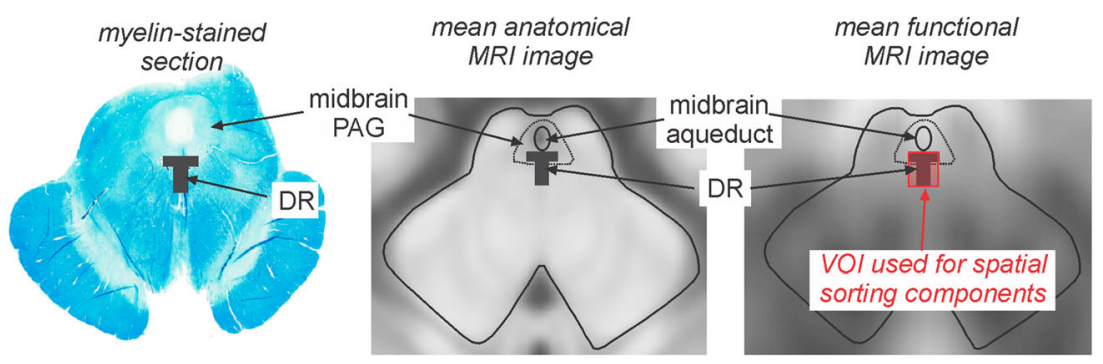

Fig. 1 Axial sections through the midbrain outlining the location of the dorsal raphe nucleus (DR) and the volume of interest (VOI) used to spatially sort the individual-component signal intensity changes. On the left is a myelin-stained image, in the middle a mean T1-weighted anatomical magnetic resonance imaging (MRI) image, and on the right a mean functional MRI image averaged from all 62 subjects. The dotted line indicates the approximate boundaries of the midbrain periaqueductal gray (PAG). The red shading indicates the location of the VOI used for spatially sorting the signal components 


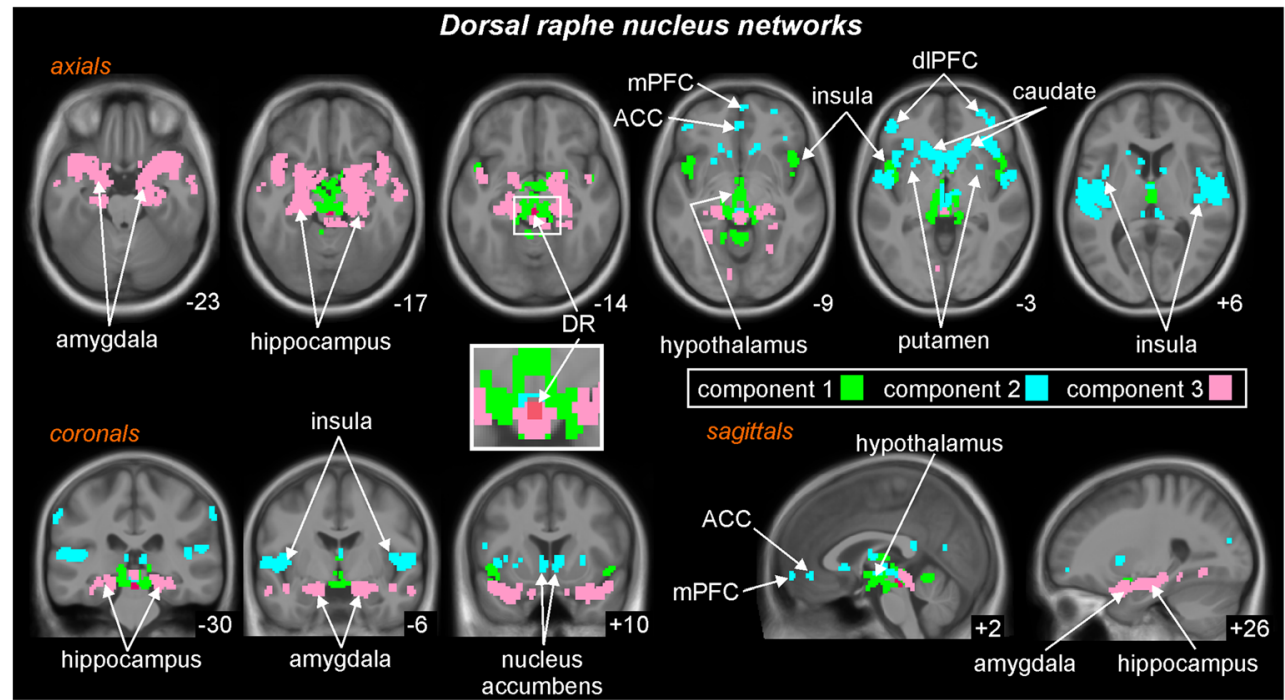

Fig. 2 Three separate signal intensity change pattern maps (Components 1,2 , and 3), each including the dorsal raphe nucleus (DR) overlaid onto a mean T1-weighted anatomical image set. Note that signal intensity within the DR covaried with the signal intensities within the amygdala, hippocampus, hypothalamus, anterior cingulate cortex (ACC), medial

pole. Together these three components, which all included the region of the DR, encompassed the majority of the ascending serotonergic system.

\section{Dorsal raphe network and harm avoidance}

Assessing the influence of HA on resting-signal intensity fluctuations resulted in significant relationships in a number of discrete regions (Figs. 3 and 4, Table 1). These regions included bilateral insula, nucleus accumbens, ventral hippocampus, the basal nucleus of the amygdala, and mPFC. Interestingly, in some regions there appeared to be a lateralization effect with respect to the direction of these relationships. Signal intensity fluctuation magnitudes in the left amygdala were negatively correlated with HA $(r=-.46, p=$ .0002), whereas signal fluctuations within the right amygdala were positively correlated with HA $(r=.37$, $p=.003)$. Similarly, signal fluctuations within the left insula were negatively correlated with HA $(r=-.3, p=.016)$, but within the right insula they were positively correlated $(r=.25$, $p=.046)$. Conversely, the signal in the left ventral hippocampus displayed a positive relationship with HA $(r=.36, p=.004)$, and the signal within the right ventral hippocampus a negative relationship $(r=-.41, p=.0009)$. The mPFC also displayed both positive and negative correlations with HA (Component 2, $r=-.37, p=.003$; Component 3, $r=.32, p=.01)$. Finally, both the right and left nucleus accumbens displayed negative relationships with HA (left, $r=-.37, p=.003$; right, $r=-.39, p=.002$ ), and the DR, a positive relationship $(r=.37, p=.003)$. prefrontal cortex (mPFC), dorsolateral prefrontal cortex (dlPFC), insula, and nucleus accumbens. Slice locations in Montreal Neurological Institute (MNI) space are indicated at the lower right of each image. The DR volume of interest used to spatially sort the signal component maps is shown in red

In addition to being significantly correlated to HA, for each of these clusters, signal intensity magnitudes were significantly different between those individuals with high HA (score $>$ 99) and those with low HA (score < 83) (mean $[ \pm S E M]$ parameter estimate values, low HA vs. high HA): left amygdala, $1.34 \pm 0.18$ vs. $0.53 \pm 0.19, p=.002$; right amygdala, $0.45 \pm 0.26$ vs. $1.68 \pm 0.36, p=.004$; left insula, $-0.13 \pm 0.15$ vs. $-0.52 \pm 0.12, p=.027$; right insula, $0.41 \pm 0.44$ vs. $1.48 \pm$ $0.31, p=.028$; left ventral hippocampus, $0.45 \pm 0.2$ vs. $0.97 \pm$ $0.16, p=.025$; right ventral hippocampus, $1.37 \pm 0.10$ vs. 0.68 $\pm 0.14, p=.0001 ; \mathrm{mPFC}$ (Component 2), $0.01 \pm 0.18$ vs. $-0.46 \pm 0.13, p=.021 ; \mathrm{mPFC}$ (Component 3), $0.80 \pm 0.19$ vs. $0.23 \pm 0.13, p=.01$; right nucleus accumbens, $1.04 \pm 0.21$ vs. $0.42 \pm 0.15, p=.01$; left nucleus accumbens, $0.84 \pm 0.12$ vs. $0.30 \pm 0.14, p=.003$; and DR, $1.52 \pm 0.21$ vs. $1.02 \pm 0.22$, $p=.04$.

\section{Discussion}

In accordance with the aim of this study, these data reveal that at rest, signal intensity fluctuations within discrete parts of DR networks are influenced by an individual's HA, which raises the possibility that ongoing activity within these regions may be involved in the expression of a particular behavioral response in relevant situations. In this investigation, we showed that DR signal intensity fluctuations covaried with the amygdala, hippocampus, insula, and nucleus accumbens, as well as the cingulate and prefrontal cortices, which is consistent with our first hypothesis. In addition, to address our second 


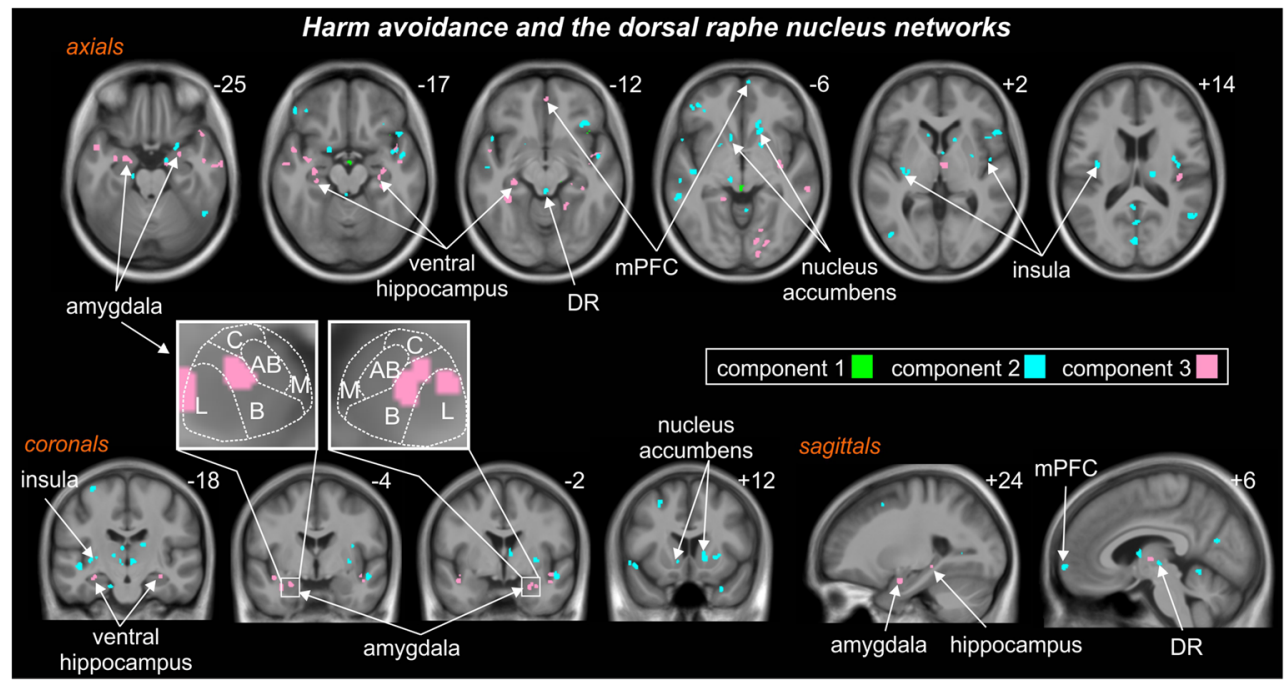

Fig. 3 Resting-signal intensity fluctuations of the previously defined networks significantly correlated to harm avoidance (HA) were overlaid on a mean T1-weighted anatomical image set. Note that resting-signal connectivity was correlated with HA in the region of the dorsal raphe nucleus (DR), the left and right amygdala in the region of the basal and basolateral nuclei, left and right ventral hippocampus, left and right nucleus accumbens, left and right insula, and medial prefrontal cortex (mPFC). This reflects areas that correlate with HA. The slice locations in Montreal Neurological Institute (MNI) space are indicated at the upper right of each image. Amygdala nuclei: $\mathrm{AB}$, accessory basal nucleus; $\mathrm{B}$, basal nucleus; $\mathrm{C}$, central nucleus; $\mathrm{L}$, lateral nucleus; $\mathrm{M}$, medial nucleus

hippocampus, insula, and nucleus accumbens, as well as the medial prefrontal cortex. These results are interesting when

considering potential roles of the serotonergic system in the hypothesis, we found that the strength of these signal intensity fluctuations was significantly correlated with HA in a number of regions, including the bilateral basal amygdala, ventral
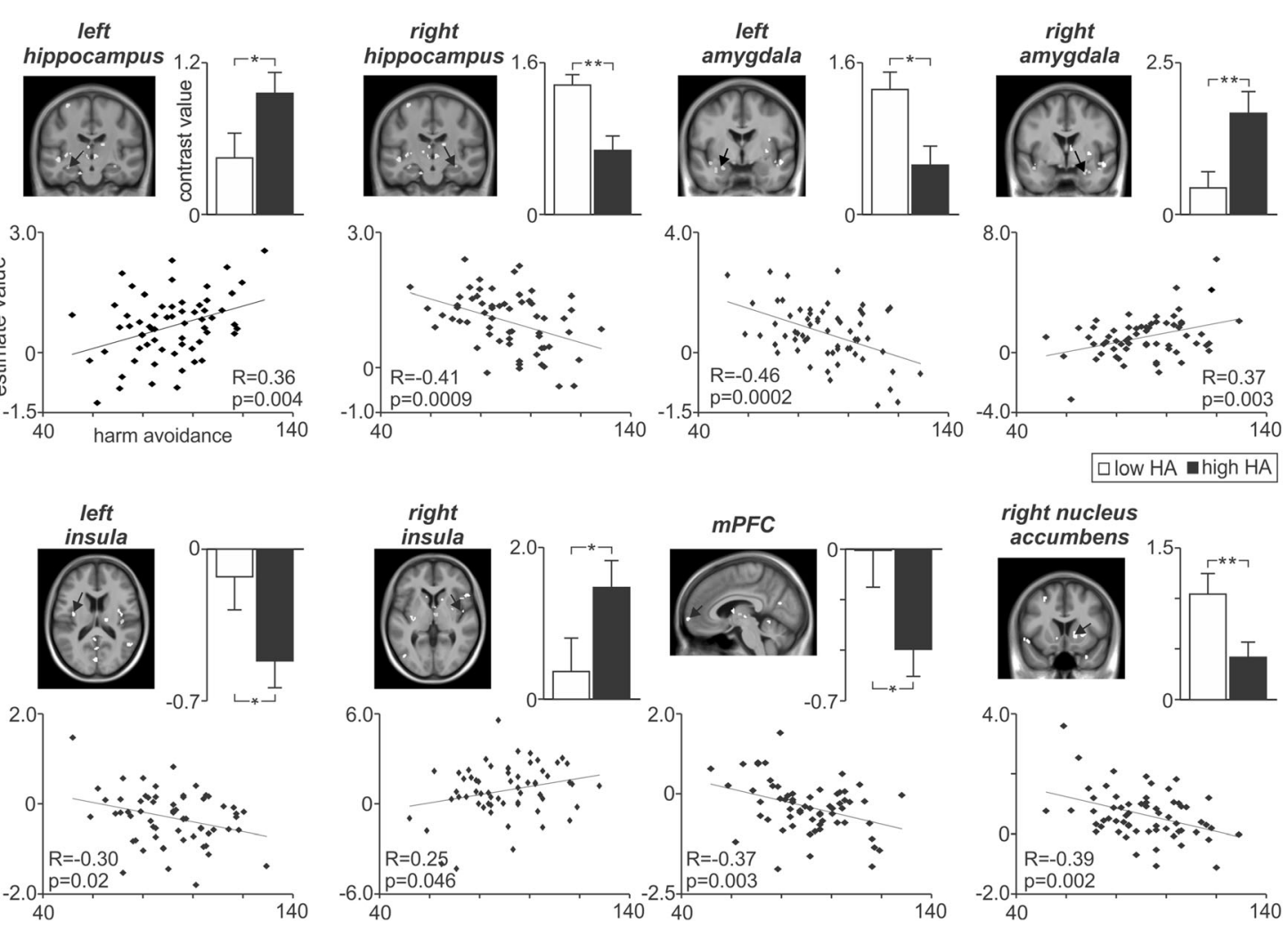

Fig. 4 Plots of harm avoidance (HA) against parameter estimate values (i.e., resting-signal connectivity strength) in significantly correlated regions. In addition to displaying significant linear relationships, in each

region the magnitudes of resting connectivity strength were significantly different in those individuals with low HA (white bars) versus those with high HA (black bars). ${ }^{*} p<.05,{ }^{* *} p<.01$. mPFC: medial prefrontal cortex 
Table 1 Brain regions in which signal intensity was significantly correlated to harm avoidance

\begin{tabular}{llllll}
\hline & \multicolumn{2}{l}{ MNI Coordinates } & \multirow{2}{*}{ Cluster Size } & $t$ Value \\
\cline { 2 - 4 } & $X$ & $Y$ & $Z$ & & \\
\hline Right amygdala & 26 & 0 & -24 & 7 & 2.57 \\
Left amygdala & 30 & -2 & -24 & 6 & 2.42 \\
Left hippocampus & -24 & -4 & -22 & 10 & 2.54 \\
& -28 & -26 & -14 & 23 & 3.62 \\
& -30 & -18 & -16 & 22 & 3.36 \\
Right hippocampus & -24 & -4 & -22 & 10 & 2.54 \\
Left insula & -32 & -6 & -24 & 8 & 2.86 \\
Right insula & -34 & -16 & -16 & 26 & 3.87 \\
Left nucleus accumbens & -10 & 16 & 0 & 11 & 3.32 \\
Right nucleus accumbens & 16 & 20 & -6 & 17 & 3.75 \\
Medial prefrontal cortex & 2 & 54 & -8 & 17 & 2.38 \\
& 8 & 62 & -4 & 6 & 3.16 \\
Dorsal raphe nucleus & 4 & -18 & -2 & 16 & 3.08 \\
\hline
\end{tabular}

expression of HA behaviors, because the networks identified are based not only on activity within and between the DR, but are also consistent with the well-known ascending serotonergic system (Vertes \& Linley, 2007).

Our ICA revealed 40 neural networks, three of which were selected for their higher overlap with the DR. ICA is a technique that decomposes a signal into independent parts, and as a consequence, it is significantly more powerful and sensitive than standard fMRI image analysis techniques (McKeown \& Sejnowski, 1998). The identification of differing signal intensity patterns within the DR networks with largely different parts of the brain highlights the importance of performing an ICA as opposed to a standard functional connectivity analysis. The latter is unable to resolve separate signal sources, and therefore much of the signal would be hidden (Frigyesi et al., 2006). Furthermore, this signal decomposition procedure allowed us to reduce the effects of extraneous parameters such as heart rate or head movement, by removing those component maps known to be sensitive to heart rate (such as those encompassing the brain ventricles), or movement (such as those comprising the edge of the brain). Following removal of these component maps, within the DR networks identified we found that HA was significantly correlated with resting activity in the basal amygdala and ventral hippocampus. This is in accordance with the Deakin and Graeff hypothesis, suggesting that serotonergic neurons in the DR project to the basolateral amygdala and ventral hippocampus in the process of mediating conflict and anxiety (Paul \& Lowry, 2013). Indeed, it is well-known that the amygdala plays a critical role in emotional regulation and anxiety, and that amygdala dysregulation predisposes an individual to developing anxiety disorders, which are associated with significantly increased HA (Hariri \& Whalen, 2011; Pessoa, 2011; Starcevic, Uhlenhuth, Fallon, \& Pathak, 1996). The amygdala has been proposed to play a key role in anxiety, due to its participation in the processing of warnings and sensitivity to punishment, its regulation of stress-related memory through induced plasticity, and its control of the fear-anxiety hierarchy (Gray \& McNaughton, 2000; McNaughton \& Corr, 2004; Roozendaal, McEwen, \& Chattarji, 2009). Furthermore, serotoninmediated amygdala activity has been implicated as being a potential pathway through which selective serotonin reuptake inhibitors (SSRIs) can achieve their anxiolytic effect (Bigos, Pollock, Aizenstein, Fisher, Bies, \& Hariri, 2008). A limitation of this study is that we could not measure serotonergic output; rather, we inferred it from signal intensity changes within the DR. Of course, this limits the inferences we can make with regard to the integration of our results with the role of serotonin in behavioral responses.

In this study, we found that the signal intensity fluctuations significantly correlated to HA were located primarily within the basal subdivision of the amygdala, which projects heavily to the nucleus accumbens and to prefrontal and hippocampal cortices (Roozendaal, McReynolds, Van der Zee, Lee, McGaugh, \& McIntyre, 2009). A significant correlation between HA and bilateral basal amygdala activity is consistent with previous investigations that have shown that amygdala responses during emotional conflict are significantly altered in individuals with generalized anxiety disorder, a condition also characterized by increased levels of HA (Etkin, Prater, Hoeft, Menon, \& Schatzberg, 2010; Starcevic et al., 1996). In addition to altered responsiveness, human positron emission tomography (PET) has revealed that HA is associated with increased resting activity in the right amygdala (Silk, 1998). This is further supported by a PET study showing that the processing of aversive emotions in the amygdala is directly connected to the availability of the serotonin receptor $5-\mathrm{HT}_{1 \mathrm{~A}}$ in the DR (Selvaraj, Mouchlianitis, Faulkner, Turkheimer, Cowen, Roiser, \& Howes, 2015). The direct link between serotonin release and amygdala activity was further demonstrated by Fisher and colleagues (Fisher, Meltzer, Ziolko, Price, Moses-Kolko, Berga, \& Hariri, 2006), who reported a significant relationship between reduced serotonin release and increased amygdala activity. These data suggest that stressful events can lead to sustained functional and anatomical changes within the basal amygdala, which may contribute to the development of anxiety disorders.

In addition to the amygdala, the hippocampus also displayed significant signal covariation correlated with HA. This HA relationship was restricted to the ventral hippocampal region, which is consistent with Deakin and Graeff's hypothesis that DR serotonergic neurons enable anxiety-like behavior through distribution to the ventral hippocampus (Paul 
\& Lowry, 2013). A view is emerging that the hippocampus is a functional heterogeneous structure along its longitudinal axis, with the dorsal hippocampus involved in learning and spatial memory, and the ventral aspect in regulating anxiety-like and emotional/motivational behaviors (Bannerman, Rawlins, McHugh, Deacon, Yee, Bast, \& Feldon, 2004; Fanselow \& Dong, 2010; Strange, Witter, Lein, \& Moser, 2014). This differentiation is supported by the finding that serotonergic fibers provide denser input to the ventral hippocampus (Kheirbek \& Hen, 2011; Tanaka, Samuels, \& Hen, 2012) and findings of strong connections between the ventral hippocampus and other brain regions known to be involved in emotional behavior regulation, such as the nucleus accumbens, prefrontal cortex, and amygdala (Bannerman et al., 2004; Sahay \& Hen, 2007). The hippocampus plays an important role in anxiety; hippocampal lesions result in behavioral disinhibition and reduced anxiety (Bannerman et al., 2004). Additionally, increased hippocampal volume has been associated with increased behavioral inhibition and increased anxiety, whereas smaller volumes have been associated with impulsiveness (Cherbuin, Windsor, Anstey, Maller, Meslin, \& Sachdev, 2008). Both the amygdala and the ventral hippocampus have been found to contribute to the processing of anxiogenic stimuli, and considering that anxiety disorders are on average accompanied by high HA (Tuominen, Salo, Hirvonen, Nagren, Laine, Melartin, \& Hietala, 2012), these studies support our findings of correlations with HA in these two regions.

We also found significant resting-activity correlations with HA in the insular cortex and the nucleus accumbens. The insula has been heavily implicated in emotion processing and anxiety, and has been proposed to be part of a network that predisposes individuals with anxiety-related temperamental traits, such as high HA, to express elevated symptoms under stress (Stein, Simmons, Feinstein, \& Paulus, 2007). Despite a lack of evidence for the role of the insula in emotional networks, apart from its role during cognitive/emotional task paradigms, PET studies have shown that HA is associated with altered baseline activity in the insula (Silk, 1998). Similarly, although the nucleus accumbens is frequently associated with evoked behaviors such as reward and is most often associated with dopaminergic activity, it does receive direct inputs from both the basal amygdala and the ventral hippocampus (Roozandaal et al, 2009; Sahay \& Hen, 2007). The nucleus accumbens has also been implicated in the fear system, with serotonergic projections from the DR innervating the nucleus accumbens and amygdala, amongst other areas of the forebrain, guiding individuals away from danger cues (Deakin \& Graeff, 1991). Indeed, nucleus accumbens activity has been associated with modulation of instrumental action involving response inhibition, with the goal of avoiding aversion and increasing chances of reward (Levita, Hoskin, \& Champi, 2012). Given this background, it is possible that the coupling between HA and activity within the nucleus accumbens is associated with an individual avoiding aversion when placed into a relevant situation.

Interestingly, the bilateral directions of the relationships between signal intensity fluctuations within the left and right amygdala, ventral hippocampus, and insula were the opposite of each other; that is, on one side they were positive, and on the other negative. Higher-order lateralization is a welldescribed phenomenon, and lateralized function has been described for many higher-order brain regions. Indeed, it was recently shown that the right amygdala displayed stronger HA-dependent resting functional connectivity with the insula, dIPFC, and orbitofrontal PFC compared to the left amygdala, whereas the left amygdala displayed greater connectivity than the right with the nucleus accumbens/subgenual cingulate cortex (Baeken, Marinazzo, Van Schuerbeek, Wu, De Mey, Bossuyt, \& De Raedt, 2014). Furthermore, during a risktaking decision-making task, it has been shown that right insula activation was significantly stronger when individuals selected a risky as compared with a safe response (Paulus, Rogalsky, Simmons, Feinstein, \& Stein, 2003). Our study also supports lateralized brain function with respect to HA and suggests that ongoing activity within the amygdala, hippocampus, and insula displays lateralized differences that may be critical when an individual is choosing an appropriate behavioral response.

Surprisingly, we did not find significant signal correlations between resting activity within the cingulate cortex and HA. This lack of a relationship at rest does not eliminate the possibility that evoked activity in the cingulate cortex is related to HA. Indeed, the ACC has been found to be involved in the control of active avoidance in response to a stimulus (McNaughton \& Corr, 2004), suggesting that signal correlations within the cingulate cortex and HA would occur under a cognitive/task paradigm. Since resting signal intensity fluctuations were assessed in this study, the responsiveness of the cingulate to appropriate tasks could not be determined. Furthermore, although self-report questionnaires are efficient to administer and guarantee that all subjects receive the same questions under the same conditions, it is widely known that they can be biased, unreliable, and influenced by an individual's subjective mental state at the time (Chien \& Dunner, 1996).

One of the most effective anxiolytic compounds, SSRIs, acts on the serotonergic system, and it has been proposed that an individual's response to SSRIs can be predicted by his or her temperamental tendencies (Phan, Lee, \& Coccaro, 2011). However, the direction of the relationship between serotonin release and HA remains unresolved, with empirical research presenting very contradictory findings. For example, some evidence suggests that serotonin facilitates anticipatory worry but at the same time restrains spontaneous panic (Deakin \& Graeff, 1991). Complicating the situation is the finding that serotonin release is tightly regulated by autoreceptors that control it via negative-feedback inhibition. Indeed, increasing 
autoreceptor function is anxiolytic, which is presumably associated with reduced serotonergic function and, as our data suggest, reduced HA (McDevitt \& Neumaier, 2011). Overall, it appears that our current understanding of serotonin release, and the mechanism of action of SSRIs, is still in its infancy, and further investigations will be needed to determine the precise relationship between serotonin and HA.

\section{Conclusions}

Our results define a neural network thats activity at rest is modulated by HA in healthy individuals. Given the strong relationship between HA and anxiety-like behaviors, it is possible that the magnitude of the resting signal intensity fluctuations in these DR networks renders an individual vulnerable to developing anxiety disorders. Increasing the understanding of $\mathrm{HA}$ as a biological predisposition underlain by a DR network could lead to interesting insights into the role of the serotonergic system, especially since we found that the DR networks overlapped with regions of the ascending serotonergic system. Furthermore, establishing these networks suggests that when an individual's HA level has reached a certain threshold due to stress, the individual may be very susceptible to developing psychological symptoms.

Author note N.M. collected and analyzed all of the data, wrote the first draft of the manuscript, altered it following feedback, and approved the final manuscript. L.A.H. designed the study, collected the data, directed the analysis and manuscript writing, and approved the final manuscript. The authors have no conflicts of interest. This research was supported by the National Health and Medical Research Council of Australia, Grant No. 1032072.

\section{References}

Arnold, P. D., Zai, G., \& Richter, M. A. (2004). Genetics of anxiety disorders. Current Psychiatry Reports, 6, 243-254.

Baeken, C., De Raedt, R., Ramsey, N., Van Schuerbeek, P., Hermes, D., Bossuyt, A., \& Luypaert, R. (2009). Amygdala responses to positively and negatively valenced baby faces in healthy female volunteers: Influences of individual differences in harm avoidance. Brain Research, 1296, 94-103. doi:10.1016/j.brainres.2009.08.010

Baeken, C., Marinazzo, D., Van Schuerbeek, P., Wu, G. R., De Mey, J., Luypaert, R., \& De Raedt, R. (2014). Left and right amygdalaMediofrontal cortical functional connectivity is differentially modulated by harm avoidance. PLOS ONE, 9, e95740. doi:10.1371/ journal.pone. 0095740

Baker, K. G., Halliday, G. M., \& Tork, I. (1990). Cytoarchitecture of the human dorsal raphe nucleus. Journal of Comparative Neurology, 301, 147-161.

Bannerman, D. M., Rawlins, J. N., McHugh, S. B., Deacon, R. M., Yee, B. K., Bast, T., \& Feldon, J. (2004). Regional dissociations within the hippocampus-Memory and anxiety. Neuroscience \& Biobehavioral Reviews, 28, 273-283. doi:10.1016/j.neubiorev. 2004.03.004
Bigos, K. L., Pollock, B. G., Aizenstein, H. J., Fisher, P. M., Bies, R. R., \& Hariri, A. R. (2008). Acute 5-HT reuptake blockade potentiates human amygdala reactivity. Neuropsychopharmacology, 33, 32213225 .

Brown, G. D. A., Yamada, S., \& Sejnowski, T. J. (2001). Independent component analysis at the neural cocktail party. Trends in Neurosciences, 24, 54-63.

Calhoun, V. D., Adali, T., Pearlson, G. D., \& Pekar, J. J. (2001). A method for making group inferences from functional MRI data using independent component analysis. Human Brain Mapping, 14, 140-151.

Carver, C. S., \& Miller, C. J. (2006). Relations of serotonin function to personality: Current views and a key methodological issue. Psychiatry Research, 144, 1-15. doi:10.1016/j.psychres.2006.03. 013

Cherbuin, N., Windsor, T. D., Anstey, K. J., Maller, J. J., Meslin, C., \& Sachdev, P. S. (2008). Hippocampal volume is positively associated with behavioural inhibition (BIS) in a large community-based sample of mid-life adults: The PATH through life study. Social Cognitive and Affective Neuroscience, 3, 262-269.

Chien, A. J., \& Dunner, D. L. (1996). The Tridimensional Personality Questionnaire in depression: State versus trait issues. Journal of Psychiatric Research, 30, 21-27.

Cloninger, C. R. (1986). A unified biosocial theory of personality and its role in the development of anxiety states. Psychiatric Developments, 4, 167-226.

Cloninger, C., Svrakic, D. M., \& Przybeck, T. R. (1993). A psychobiological model of temperament and character. Archives of General Psychiatry, 50, 975-990.

Commons, K. G., Connolley, K. R., \& Valentino, R. J. (2003). A neurochemically distinct dorsal raphe-limbic circuit with a potential role in affective disorders. Neuropsychopharmacology, 28, 206215.

Deakin, J. F., \& Graeff, F. G. (1991). 5-HT and mechanisms of defence. Journal of Psychopharmacology, 5, 305-315.

Duvernoy, H. M. (1995). The human brain stem and cerebellum: Surface, structure, vascularization, and three-dimensional sectional anatomy with MRI. New York: Springer.

Etkin, A., Prater, K. E., Hoeft, F., Menon, V., \& Schatzberg, A. F. (2010). Failure of anterior cingulate activation and connectivity with the amygdala during implicit regulation of emotional processing in generalized anxiety disorder. American Journal of Psychiatry, 167, 545-554.

Fanselow, M. S., \& Dong, H. W. (2010). Are the dorsal and ventral hippocampus functionally distinct structures? Neuron, 65, 7-19.

Fisher, P. M., Meltzer, C. C., Ziolko, S. K., Price, J. C., Moses-Kolko, E. L., Berga, S. L., \& Hariri, A. R. (2006). Capacity for 5-HT1Amediated autoregulation predicts amygdala reactivity. Nature Neuroscience, 9, 1362-1363.

Frigyesi, A., Veerla, S., Lindgren, D., \& Höglund, M. (2006). Independent component analysis reveals new and biologically significant structures in micro array data. BMC Bioinformatics, 7, 290. doi:10.1186/1471-2105-7-290

Friston, K. J., Holmes, A. P., Poline, J. B., Grasby, P. J., Williams, S. C., Frackowiak, R. S., \& Turner, R. (1995). Analysis of fMRI timeseries revisited. NeuroImage, 2, 45-53.

Gray, J. A., \& McNaughton, N. J. (2000). The neuropsychology of anxiety. Oxford: Oxford Medical.

Hariri, A. R., \& Whalen, P. J. (2011). The amygdala: Inside and out. F1000 Reports: Biology, 3, 2.

Kenna, G. A., Roder-Hanna, N., Leggio, L., Zywiak, W. H., Clifford, J., Edwards, S., \& Swift, R. M. (2012). Association of the 5-HTT genelinked promoter region (5-HTTLPR) polymorphism with psychiatric disorders: Review of psychopathology and pharmacotherapy. Journal of Pharmacogenomics and Personalized Medicine, 5, 1935. doi:10.2147/PGPM.S23462 
Kheirbek, M. A., \& Hen, R. (2011). Dorsal vs. ventral hippocampal neurogenesis: Implications for cognition and mood. Neuropsychopharmacology, 36, 373-374.

Levita, L., Hoskin, R., \& Champi, S. (2012). Avoidance of harm and anxiety: A role for the nucleus accumbens. NeuroImage, 62, 189 198. doi:10.1016/j.neuroimage.2012.04.059

Lovinger, D. M. (1999). The role of serotonin in alcohol's effects on the brain. Current Separations, 18, 23-28.

Macey, P. M., Macey, K. E., Kumar, R., \& Harper, R. M. (2004). A method for removal of global effects from fMRI time series. NeuroImage, 22, 360-366.

McDevitt, R. A., \& Neumaier, J. F. (2011). Regulation of dorsal raphe nucleus function by serotonin autoreceptors: A behavioral perspective. Journal of Chemical Neuroanatomy, 41, 234-246.

McKeown, M. J., \& Sejnowski, T. J. (1998). Independent component analysis of fMRI data: Examining the assumptions. Human Brain Mapping, 6, 368-372.

McNaughton, N., \& Corr, P. J. (2004). A two-dimensional neuropsychology of defense: Fear/anxiety and defensive distance. Neuroscience \& Biobehavioral Reviews, 28, 285-305. doi:10.1016/j.neubiorev. 2004.03.005

Montag, C., Reuter, M., Jurkiewicz, M., Markett, S., \& Panksepp, J. (2013). Imaging the structure of the human anxious brain: A review of findings from neuroscientific personality psychology. Reviews in the Neurosciences, 24, 167-190.

Paul, E. D., \& Lowry, C. A. (2013). Functional topography of serotonergic systems supports the Deakin/Graeff hypothesis of anxiety and affective disorders. Journal of Psychopharmacology, 27, 1090 1106. doi: $10.1177 / 0269881113490328$

Paulus, M. P., Rogalsky, C., Simmons, A., Feinstein, J. S., \& Stein, M. B. (2003). Increased activation in the right insula during risk-taking decision making is related to harm avoidance and neuroticism. NeuroImage, 19, 1439-1448.

Peirson, A. R., Heuchert, J. W., Thomala, L., Berk, M., Plein, H., \& Cloninger, C. R. (1999). Relationship between serotonin and the temperament and character inventory. Psychiatry Research, 89, 29-37.

Pessoa, L. (2011). Emotion and cognition and the amygdala: From "what is it?" to "what's to be done?". Neuropsychologia, 49, 681-694. doi: 10.1016/j.neuropsychologia.2011.02.030

Phan, K. L., Lee, R., \& Coccaro, E. F. (2011). Personality predictors of antiaggressive response to fluoxetine: Inverse association with neuroticism and harm avoidance. International Clinical Psychopharmacology, 26, 278-283.

Roozendaal, B., McEwen, B. S., \& Chattarji, S. (2009). Stress, memory and the amygdala. Nature Reviews Neuroscience, 10, 423-433.
Roozendaal, B., McReynolds, J. R., Van der Zee, E. A., Lee, S., McGaugh, J. L., \& McIntyre, C. K. (2009). Glucocorticoid effects on memory consolidation depend on functional interactions between the medial prefrontal cortex and basolateral amygdala. Journal of Neuroscience, 29, 14299-14308. doi:10.1523/JNEUROSCI.362609.2009

Sahay, A., \& Hen, R. (2007). Adult hippocampal neurogenesis in depression. Nature Neuroscience, 10, 1110-1115.

Selvaraj, S., Mouchlianitis, E., Faulkner, P., Turkheimer, F., Cowen, P. J., Roiser, J. P., \& Howes, O. (2015). Presynaptic serotoninergic regulation of emotional processing: A multimodal brain imaging study. Biological Psychiatry, 78, 563-571. doi:10.1016/j.biopsych.2014. 04.011

Silk, K. R. (1998). Biology of personality disorders. Washington: American Psychiatric Press.

Starcevic, V., Uhlenhuth, E. H., Fallon, S., \& Pathak, D. (1996). Personality dimensions in panic disorder and generalized anxiety disorder. Journal of Affective Disorders, 37, 75-79.

Stein, M. B., Simmons, A. N., Feinstein, J. S., \& Paulus, M. P. (2007). Increased amygdala and insula activation during emotion processing in anxiety-prone subjects. American Journal of Psychiatry, 164, 318-327.

Strange, B. A., Witter, M. P., Lein, E. S., \& Moser, E. I. (2014). Functional organization of the hippocampal longitudinal axis. Nature Reviews Neuroscience, 15, 655-669.

Tanaka, K. F., Samuels, B. A., \& Hen, R. (2012). Serotonin receptor expression along the dorsal-ventral axis of mouse hippocampus. Philosophical Transactions of the Royal Society, 367, 2395-2401. doi:10.1098/rstb.2012.0038

Tuominen, L., Salo, J., Hirvonen, J., Nagren, K., Laine, P., Melartin, T., \& Hietala, J. (2012). Temperament trait harm avoidance associates with mu-opioid receptor availability in frontal cortex: A PET study using [(11)C]carfentanil. NeuroImage, 61, 670-676.

Vertes, R. P., \& Linley, S. B. (2007). Comparison of projections of the dorsal and median raphe nuclei, with some functional considerations. International Congress Series, 1304, 98-120.

Waselus, M., Valentino, R. J., \& Van Bockstaele, E. J. (2011). Collateralized dorsal raphe nucleus projections: A mechanism for the integration of diverse functions during stress. Journal of Chemical Neuroanatomy, 41, 266-280.

Westlye, L. T., Bjornebekk, A., Grydeland, H., Fjell, A. M., \& Walhovd, K. B. (2011). Linking an anxiety-related personality trait to brain white matter microstructure: Diffusion tensor imaging and harm avoidance. Archives of General Psychiatry, 68, 369-377. doi:10. 1001/archgenpsychiatry.2011.24 